\title{
The reviewers: a critical viewpoint
}

\author{
Maura Da Cunha \\ $A B B$, Editor of Structural Botany \\ Universidade Estadual do Norte Fluminense Darcy Ribeiro
}

How many manuscripts have you received to review? How many for national journals? How many for international journals? What do you gain in return for this type of work? Have you ever stopped to think about it? In this article I discuss the role of researchers within the editorial system, and the evaluation of manuscripts for national and international journals.

Every time an author submits a manuscript the editor-in-chief or associate editor must review the submission to make sure the manuscript is properly formatted and the subject matter fits within the aim and scope of the journal. If this is done the system does not overburden the reviewers with tasks that are the journal's responsibility. This process is clearly used by many international journals but has not always been followed by journals in Brazil. Why? We need to be more disciplined to increase the quality of the journals in this country. To review a manuscript that is poorly written and does not follow the journal's format is discouraging to anyone. We should think of ourselves as reviewers and as authors.

We also need to reject manuscripts when their scientific expectations are not followed. This seems obvious but it is not always an easy task. The reasons to reject an article for publication can be objective when journal instructions are not followed or implicit based on previous articles published by the journal. Most authors understand the focus of each journal, which helps them decide where to submit an article. However, it is also necessary to reject manuscripts that will not contribute relevant information to the field, and, therefore, will not be cited by others. In addition, we should encourage authors to exclude unnecessary text and images; Brazilians have a tendency to duplicate information (e.g., the same data is presented in more than one figure and the text). Avoiding this problem will result in more concise text, which is a characteristic of scientific writing.

Several journals solicit reviewers based on their curriculum vitae, published papers, and expertise. For Brazilian journals, the criteria to choose reviewers may be more rigorous because in general we know the authors and in several situations they are colleagues that work on the same subject. This makes the process difficult for the editor-in-chief and associate editors, as is the case for Acta Botanica Brasilica.
Why don't we send manuscripts to reviewers outside Brazil? The language is the limitation. Most of the manuscripts submitted to Brazilian journals are in Portuguese. What about the manuscripts that are submitted in English? This is also a problem for the editors because it is often difficult to find someone outside of the country that is an expert in the subject of the article when you don't deal with the specific subject of the manuscript.

Overall, we rely on the goodwill of Brazilian reviewers. Should we really call it goodwill? Many times, yes. I would like to talk about the role that a reviewer/researcher has. I know some will not agree, but to be a reviewer for a national or international journal is important because carefully reviewed manuscripts help improve the quality of journals. If one always refuses to review manuscripts it will be hard to improve Brazilian journals. We need notorious botanists active in the editorial system, reviewing manuscripts and teaching young researchers how to do the same.

How do we teach young researchers to be good reviewers? This is another problem in Brazil. There are young reviewers (and some not so young) that have good curricula vitae but have not been instructed on how to review a manuscript. Some mock, are ironic, or make fun of the text in their reviews. How can a journal send such a review to an author? I was not taught how to write a review, but I did learn that we should be responsible and professional. On the other hand, we should value young researchers, many times indicated by their former professors, who are motivated and give consistent, professional reviews.

Another subject to be discussed is the time it takes to get a response. When we add to our curricula vitae that we are reviewers for certain journals, we should follow the rules of these publications. We know that there are delays within this process, such as how long it takes an author to return a manuscript after it has been reviewed. We need to have a deadline for the authors to return the corrected version and, with no mercy, refuse to publish an article if the manuscript is not returned on time. We should also take into consideration the time it takes a reviewer to return a manuscript. If the editor asks for four weeks we should return the manuscript within this time or decline to review it. In the last case, we are back to the goodwill of some researchers. 
Do our journals only need the goodwill of some? I ask you, if your manuscript, project, or scientific report needs to be reviewed, is it fair to count on the goodwill of only a few of us?

Also, how do we judge ethics and conflict of interest? Meanwhile, for the international journals we do our best, even with difficulties regarding language, sending consistent reviews before deadlines that help improve the impact factors of these journals. On the other hand, we continue to defame the national journals by sending our best works to foreign journals, leaving the least important papers to be published here.

To finish, I remind you that these are not the only reasons that limit the improvement of Brazilian journals, but they represent part of the immaturity of our national publications, which is a reflection of our own immaturity as researchers. 